\title{
Electrosorption at metal surfaces from first principles
}

\author{
Nicolas G. Hörmann $\mathbb{D}^{1,2 凶}$, Nicola Marzari ${ }^{2}$ and Karsten Reuter ${ }^{1,3}$
}

Electrosorption of solvated species at metal electrodes is a most fundamental class of processes in interfacial electrochemistry. Here, we use its sensitive dependence on the electric double layer to assess the performance of ab initio thermodynamics approaches increasingly used for the first-principles description of electrocatalysis. We show analytically that computational hydrogen electrode calculations at zero net-charge can be understood as a first-order approximation to a fully grand canonical approach. Notably, higher-order terms in the applied potential caused by the charging of the double layer include contributions from adsorbate-induced changes in the work function and in the interfacial capacitance. These contributions are essential to yield prominent electrochemical phenomena such as non-Nernstian shifts of electrosorption peaks and non-integer electrosorption valencies. We illustrate this by calculating peak shifts for $\mathrm{H}$ on Pt electrodes and electrosorption valencies of halide ions on $\mathrm{Ag}$ electrodes, obtaining qualitative agreement with experimental data already when considering only second order terms. The results demonstrate the agreement between classical electrochemistry concepts and a first-principles fully grand canonical description of electrified interfaces and shed new light on the widespread computational hydrogen electrode approach.

npj Computational Materials (2020)6:136; https://doi.org/10.1038/s41524-020-00394-4

\section{INTRODUCTION}

In recent years, calculations based on ab initio thermodynamics have increasingly contributed to unraveling key processes in interfacial electrochemistry; e.g., in batteries, fuel cells, and other electrocatalytic systems. In such studies, the electrochemical environment and operation conditions are suitably represented in the form of thermodynamic reservoirs ${ }^{1-5}$. Within a grandcanonical setup these reservoirs are then coupled to predictivequality (typically density-functional theory (DFT)) total energy calculations for the electrode, to deduce electrochemical stabilities and activities; see e.g., refs. ${ }^{2,6-11}$. In the application to interfacial electrochemistry a key challenge to this general $a b$ initio thermodynamics concept is the necessity to exchange electrons with a reservoir representing the electrode potential. In principle, this requires to perform DFT calculations in various charge states. This clashes with the common representation of the electrode as finite slab using periodic-boundary conditions, for which straightforward calculations can only be performed at zero total charge of the cell. For this reason, in most practical application, the early computational hydrogen electrode (CHE) approach ${ }^{2,6,12}$ relies solely on the energetics of charge-neutral electrode calculations, in the absence of the electrochemical double layer. The dependence on the electrode potential is then included in the analysis as an a posteriori shift of the electrochemical potential of the electrons taken from the reservoir, whose number is a priori fixed according to the charge-neutrality condition.

Conceptually, this restriction can be readily lifted through the introduction of meaningful counter-charge models in the vacuum region between the periodic slabs ${ }^{1,13}$. This then allows for a surface polarization and charging of the electrode slab while still maintaining the overall charge neutrality of the supercell. In the recent fully grand-canonical (FGC) approaches this is realized in practice by the use of an implicit solvation model in the DFT calculations $s^{5,9,11,14-34}$, which captures the effects of both electrolyte polarization and solvent screening, and renders the calculations largely insensitive to details of the counter-charge model employed ${ }^{35}$ at conventional thicknesses of the vacuum/ dielectric region ${ }^{32}$. Within this approach, variable-charge calculations can nowadays be performed at high computational efficiency in a number of major DFT codes, e.g., ENVIRON ${ }^{18,36}$, VASPsol $^{21}$, FHI-aims ${ }^{25}$, Jaguar ${ }^{29}$, BigDFT $^{24}, \mathrm{GPAW}^{31,37}$, or JDFTx ${ }^{38}$ '.

The capabilities of FGC approaches have been highlighted in several publications and include a potential-dependence of chemical reaction steps ${ }^{8,29-31,39,40}$, potential-induced surface reconstructions or shifts of stable adsorption sites ${ }^{5,11,13,41}$. However, there is presently still limited understanding of the methodological differences between the still prevalent zerocharge CHE and the variable-charge FGC approaches. In this work we therefore present a consistent thermodynamic framework to compare both types of approaches and highlight the simplifications made in practical calculations. In particular, we show by analysis of a generic second-order Taylor expansion of the interface energies with respect to surface charge $7,11,20,34,39,42-45$ that the CHE approach can be understood as a first-order approximation to the FGC energetics, while to second order, double-layer charging is represented by changes in work function and interfacial capacitance.

We illustrate these differences between $\mathrm{CHE}$, second-order expansion and FGC in the application to two classic probes of interfacial electrochemistry at metal electrodes, namely nonNernstian shifts of proton electrosorption peaks at low-index $\mathrm{Pt}$ crystals $^{5,46-50}$ and the non-integer electrosorption valency of halide ions at $\mathrm{Ag}(111)^{51-54}$. While neither of these observations can be reproduced at the $\mathrm{CHE}$ level, already the second-order expansion introduces them qualitatively, if not even in quantitative agreement with existing experimental data. We find that with current simple implicit solvation models higher-order terms captured in the FGC approach do not generally lead to further significant improvements. Apart from the intrinsic shortcomings of the continuum solvation itself ${ }^{55}$, we also discuss alternative

\footnotetext{
${ }^{1}$ Chair of Theoretical Chemistry and Catalysis Research Center, Technische Universität München, Garching 85748 , Germany. ${ }^{2}$ Theory and Simulation of Materials (THEOS), and National Centre for Computational Design and Discovery of Novel Materials (MARVEL), École Polytechnique Fédérale de Lausanne, Lausanne 1015, Switzerland. ${ }^{3}$ Fritz-HaberInstitut der Max-Planck-Gesellschaft, Faradayweg 4-6, Berlin 14195, Germany. ${ }^{\bowtie}$ email: nicolas.hoermann@tum.de
} 
sources for the remaining discrepancies with experiments. As an important corollary, the present work also demonstrates analytically that the FGC energetics does not tend toward CHE results in the limit of single adsorbates in infinitely large surface unit-cells. Instead, the discussed applications suggest that second-order calculations are a computationally efficient approximation to FGC calculations.

\section{RESULTS}

$\mathrm{Ab}$ initio thermodynamics and interfacial free energy

The central quantity in our ab initio thermodynamics approach to surface electrochemistry is the Gibbs excess energy of interface configuration $a^{4,5,13}$,

$$
\begin{aligned}
G_{\text {exc }}^{a}\left(T, p, \mu_{\mathrm{s}}, \tilde{\mu}_{\mathrm{a}}, \Phi_{E}\right)= & G_{\text {surf }}^{a}\left(T, p, N_{\mathrm{s}}^{a}, N_{\mathrm{a}}^{a}, N_{e}^{\mathrm{abs}, a}\right)-N_{\mathrm{s}}^{a} \mu_{\mathrm{s}} \\
& -N_{\mathrm{a}}^{a} \tilde{\mu}_{\mathrm{a}}+N_{e}^{\mathrm{abs}, a} e \Phi_{E} .
\end{aligned}
$$

The specific interface configuration $a$ is hereby characterized by its surface geometry (e.g., the position of adsorbates) and chemical composition. In this work we will focus on adsorbates at metal electrodes and we will specify this composition in terms of the number of neutral substrate atoms $N_{\mathrm{s}}^{a}$, the number of (possibly charged) adsorbate species $N_{a}^{a}$, and the number of excess electrons $N_{e}^{\text {abs, } a}$ (conveniently measured as absolute surface charge, cf. below). The additional dependence on temperature $T$ and pressure $p$ in the grand-canonical setup is henceforth dropped for ease of notation. Appropriately normalized to the surface area $A$, the intensive interface excess free energy is

$\gamma_{\mathrm{exc}}^{a}\left(\mu_{\mathrm{s}}, \tilde{\mu}_{\mathrm{a}}, \Phi_{E}\right)=\frac{1}{A} G_{\mathrm{exc}}^{a}\left(\mu_{\mathrm{s}}, \tilde{\mu}_{\mathrm{a}}, \Phi_{E}\right)$,

and denotes the cost of creating the electrode surface $a$ in the electrochemical environment at the applied potential $\Phi_{E}$, as it compares the Gibbs free energy of the electrode $G_{\text {surf }}^{a}$ with the sum of the free energies of its constituents in their corresponding (bulk) reservoirs. These free energies are defined by the bulk chemical potential $\mu_{\mathrm{s}}$ of the (neutral) substrate atoms and the electrochemical potentials (highlighted with a tilde) of the (charged) adsorbate species $\tilde{\mu}_{\mathrm{a}}$ and of the excess electrons $\tilde{\mu}_{e}$. The latter relates to the applied electrode potential via $\tilde{\mu}_{e}=-e \Phi_{E}$. Without loss of generality, we choose the extensive Gibbs excess energies for the entire electrode as central base quantities in this work, and will therefore explicitly include a surface area dependence in the equations wherever required.

Note that the sign convention followed in Eq. (1) considers negative Gibbs free energies and (electro)chemical potentials, with more negative values corresponding to more stable or more dilute configurations. The electrode potential $\Phi_{E}$ is measured according to electrochemistry conventions, with increasing values away from the zero-reference vacuum level, such that e.g., the experimental standard hydrogen electrode (SHE) lies at $+4.44 \mathrm{~V}$ on this absolute scale ${ }^{56}$. In multicomponent systems different neutral and charged species may exist and appropriate sums over the indices $s$ and a with appropriate stoichiometries need to be introduced in Eq. (1). We stress that this need extends also generally to all interfacial species in the double layer, such as water or solvated ions ${ }^{57-59}$. However, in this work we restrict ourselves to implicit solvation models in which only the specifically bound ionic adsorbates a are treated explicitly in Eq. (1).

It is important to point out that the so-defined excess energies have often no direct thermodynamic relevance, as they still depend on the specific interface configuration $a$. The true thermodynamic excess energy $G_{\text {exc }}$ corresponds to the minimum $G_{\text {exc }}^{a}$ in the space of all possible interface configurations $a^{5}$, and this then also defines the true ground-state electrode configuration in terms of $N_{s}, N_{a}$, and $N_{e}^{\text {abs }}$. We note in passing that the two mathematical steps of formulating the excess energies $G_{\text {exc }}^{a}$ as in
Eq. (1) and then minimizing with respect to configurations $a$ correspond to a Legendre transform, which is why $G_{\text {exc }}$ approximates in fact the grand canonical free energy of the system. In a comprehensive all-explicit simulation the optimum interface configuration that defines $G_{\text {exc }}$ would eventually emerge directly from an appropriate, ideally exhaustive sampling of all corresponding degrees of freedom. In practice, $G_{\text {exc }}$ is instead often approximately determined by computing $G_{\text {exc }}^{a}$ for a set of candidate configurations $a$ with fixed $N_{\mathrm{s}}^{a}, N_{\mathrm{a}}^{a}, N_{e}^{\text {abs }, a}$, and the configuration with minimal $G_{\text {exc }}^{a}$ is declared to be the most stable one $^{3-5,60,61}$. It is also possible to compare candidate configurations and excess energies that already minimize $G_{\text {exc }}^{a}$ in a sub-space of all interface configurations $a$. In the electrochemical context, this refers notably to charge-equilibrated excess energies $\mathcal{G}_{\text {exc }}^{a}$ that minimize $G_{\text {exc }}^{a}$ with respect to the number of electronic charges $N_{e}^{\mathrm{abs}, a}$ for a configuration $a$ with defined $N_{\mathrm{s}}^{a}$ and $N_{\mathrm{a}}^{a}$

$\mathcal{G}_{\text {exc }}^{a}\left(\mu_{\mathrm{s}}, \tilde{\mu}_{\mathrm{a}}, \Phi_{E}\right)=\min _{N_{e}^{\text {abs }, \alpha}} G_{\text {exc }}^{a}\left(\mu_{\mathrm{s}}, \tilde{\mu}_{\mathrm{a}}, \Phi_{E}\right)$.

$\mathcal{G}_{\text {exc }}^{\alpha}$ then defines the cost of creating an interface with composition $N_{\mathrm{s}}^{a}$ and $N_{\mathrm{a}}^{a}$ at a given applied potential $\Phi_{E}$, as would be obtained equivalently in a constant potential calculation ${ }^{27,30,62}$.

As common in ab initio thermodynamics approaches to surface systems ${ }^{4}$, the difference in the solid-state terms in Eq. (1), i.e., $G_{\text {surf }}^{a}$ and any bulk-like reservoir $\mu_{\mathrm{s}}$, is generally approximated as

$$
\begin{aligned}
& G_{\text {surf }}^{a}\left(N_{\mathrm{s}}^{a}, N_{\mathrm{a}}^{a}, N_{e}^{\mathrm{abs}, a}\right)-N_{\mathrm{s}}^{a} \mu_{\mathrm{s}} \\
& \approx E_{\text {surf }}^{\mathrm{DFT}, a}\left(N_{\mathrm{s}}^{a}, N_{\mathrm{a}}^{a}, N_{e}^{\mathrm{abs}, a}\right)-N_{\mathrm{s}}^{a} E_{\text {bulk }, \mathrm{s}}^{\mathrm{DFT}}+\Delta F^{\mathrm{corr}, a} .
\end{aligned}
$$

Here, $E_{\text {surf }}^{\mathrm{DFT}, a}$ is the total energy of candidate structure $a$ and $E_{\text {bulk,s }}^{\mathrm{DFT}}$ is the total energy per atom of the bulk-like reservoir of neutral species $s$, both total energies being typically calculated with DFT. $\Delta F^{\mathrm{corr}, a}$ is a correction term due to the changes in the vibrational and configurational degrees of freedom of the adsorbates in candidate structure $a$ as compared to their corresponding reservoir. This free energy term is commonly assumed to be independent of the charge state $N_{e}^{\mathrm{abs}, a}$ of the candidate structure. One should note that even though $E_{\text {surf }}^{\mathrm{DFT}, a}$ is denoted here as a total energy, it does contain free energy contributions due to solvent screening and electrolyte response when an implicit solvation model is employed in the DFT calculation ${ }^{5,32}$.

\section{FGC calculations}

In Eq. (1) the absolute surface charge $N_{e}^{\text {abs }, a}$ of candidate configuration a measures the number of electrons in excess or deficient with respect to the number of electrons of the corresponding charge-neutral electrode surface at the applied potential (other references are possible without affecting the interpretation $\left.{ }^{5}\right) \cdot N_{e}^{\text {abs, } a}$ is thus the number of electrons that need to be exchanged with the external electron reservoir. In the present application to adsorbates at metal electrodes

$N_{e}^{\mathrm{abs}, a}=\frac{q_{\mathrm{a}}}{e} N_{\mathrm{a}}^{a}+N_{e}^{\mathrm{net}, a}$,

i.e., $-e N_{e}^{\text {abs }, a}$ is the sum of charges to compensate for the ion charges $q_{\mathrm{a}} N_{\mathrm{a}}^{a}$ of all adsorbates of charge $q_{\mathrm{a}}$ and the net electronic surface excess charge $-e N_{e}^{\text {net, } \alpha}$ that is itself compensated exactly by the electrolyte counter charges in the diffuse layer. Without meaningful counter-charge model, any net charge of a metallic surface poses a significant challenge in calculations with periodic boundary conditions ${ }^{63}$. In its practical realization, without explicitly represented electrolyte ions (c.f. ref. ${ }^{64}$ ) the $\mathrm{CHE}^{2,6,61}$ approach therefore considers only charge-neutral candidate structures with $N_{e}^{\text {net, } a}=0$. In order to do so, it thus assumes that each ion of charge $q_{\mathrm{a}}$ also drags $q_{\mathrm{a}}$ /e electrons onto the surface upon adsorption. Any electrochemical reaction involving a proton transfer would then, for instance, necessarily become a protoncoupled electron transfer (PCET). For such charge-neutral 
structures, solvation effects, at least on the level of implicit solvation models, are often small. This is why corresponding $\mathrm{CHE}$ work is often also based on DFT calculations performed without any solvation treatment at all.

FGC simulations in implicit solvation models instead bypass the overall supercell charge neutrality restriction by balancing any $N_{e}^{\text {net }, a} \neq 0$ through added electrolyte counter charges in the dielectric region between the periodically repeating slabs. At sufficiently high electrolyte concentrations, the details of the electrolyte model are thereby largely irrelevant for the overall energetics $5,19,32,35,39$. Practical calculations then perform the minimization of $G_{\text {exc }}^{a}$ in Eq. (1) with respect to $N_{e}^{\mathrm{abs}, a}$ for every candidate structure by explicitly calculating a number of finite charge states. Within the approximation in Eq. (4) and assuming a symmetric slab setup the minimization of Eq. (3) yields the condition

$$
\frac{\partial G_{\mathrm{exc}}^{a}\left(N_{e}^{\mathrm{abs}, a}\right)}{\partial N_{e}^{\mathrm{abs}, a}}=0 \leftrightarrow \underbrace{\frac{\partial E_{\text {surf }}^{\mathrm{DFT}, a}\left(N_{e}^{\mathrm{abs}, a}\right)}{\partial N_{e}^{\mathrm{net}, a}}}_{-e \Phi^{a}}=-e \Phi_{E},
$$

where the underbraced equality arises from Janak's theorem and the employed reference for the electrostatic potential (see Methods). Equation (6) thus states that the minimization is equivalent to choosing a system such that its work function $e \Phi^{a}$ is equal to the externally applied (target) potential $\Phi_{E}$ times the elementary charge e. Thus, the charge-equilibrated $\mathcal{G}_{\text {exc }}^{a}$ can be directly obtained by interpolating the dependence of the energy and the charge on the work function and evaluating $G_{\text {exc }}^{a}$ for charge values where the work function corresponds to $\Phi_{E}{ }^{5}$, similar to what is done in the generalized CHE and related constantpotential approaches $27,30,62$.

Obviously, this procedure increases the computational burden, as each candidate structure has to be calculated in different charge states. On the other hand, certain electrochemical observables can be simulated that would otherwise be inaccessible within the CHE approximation. Noteworthy, these include non-linear variations of the interface energy with applied potential (cf. Lippmann equation ${ }^{5,47,65-67}$ ) potential-induced surface reconstructions $s^{5,13,41}$ that maintain the overall stoichiometry in terms of $N_{\mathrm{a}}$ and $N_{\mathrm{s}}$, and $\mathrm{pH}$-shifts of electrosorption peaks on the RHE scale as well as non-integer electrosorption valencies ${ }^{51,52,54}$, as will be explicitly illustrated here.

Grand canonical energetics within a quadratic approximation Further insight into the differences between FGC and CHE results can be gained by analyzing a second-order Taylor expansion of $E_{\text {surf }}^{\mathrm{DFT} a}$ with respect to the net electronic surface excess charge $N_{e}^{\text {net }, a 7,11,34,39,42-45}$. Within a symmetric slab setup-with a total surface area of $2 A$ and a unique electrostatic reference level in the implicit region- $E_{\text {surf }}^{\mathrm{DFT}, a}$ can be expanded around the chargeneutral point $N_{e}^{\text {net, } a}=0$ as

$$
\begin{aligned}
E_{\text {surf }}^{\mathrm{DFT}, a}\left(N_{e}^{\mathrm{abs}, a}\right)= & E_{\text {surf }, 0}^{\mathrm{DFT}, a}+\underbrace{\left.\frac{\partial E_{\text {surf }}^{\mathrm{DFT}, a}}{\partial N_{e}^{\text {net }, a}}\right|_{N_{e}^{\text {net }, a}=0}}_{-e \Phi_{0}^{a}} N_{e}^{\text {net }, a} \\
& +\frac{1}{2} \underbrace{\left.\frac{\partial^{2} E_{\text {surf }}^{\mathrm{DFT}, a}}{\left.\partial N_{e}^{\text {net }, a}\right)^{2}}\right|_{N_{e}^{\text {net }, a}=0}}_{\frac{e^{2}}{c_{0}^{a}}}\left(N_{e}^{\text {net }, a}\right)^{2}+\ldots,
\end{aligned}
$$

where $E_{\text {surf, }, 0}^{\mathrm{DFT},}$ is the total energy of the uncharged system, $\Phi_{0}^{a}$ the work function and $c_{0}^{a}$ the extensive interfacial capacitance at the potential of zero charge (PZC). $c_{0}^{a}$ relates to the more common, area-normalized capacitance $C_{0}^{a}$ measured in $\mu \mathrm{F} / \mathrm{cm}^{2}$ via $c_{0}^{a}=2 A C_{0}^{a}$. Here and in the following, a subscript o will refer to quantities determined at the PZC. The explicit dependence of the total and excess energies on $N_{\mathrm{s}}^{a}$ and $N_{\mathrm{a}}^{a}$ is dropped for ease of notation.

Within this second-order expansion, the minimization of the excess free energy of candidate structure $a$ with respect to the number of electrons can be carried out analytically following Eq. (6) to obtain

$N_{e}^{\mathrm{net}, \alpha}\left(\Phi_{E}\right)=\frac{c_{0}^{a}}{e} \cdot\left(\Phi_{0}^{a}-\Phi_{E}\right)$.

Inserting Eqs. (7) and (8) into Eq. (1) then yields the chargeequilibrated excess energy $\mathcal{G}_{\text {exc }}^{a}$ up to second-order as

$$
\begin{aligned}
& \mathcal{G}_{\text {exc }}^{a}\left(\Phi_{E}\right) \\
& \approx\left[G_{\text {surf }, 0}^{a}-N_{\mathrm{s}}^{a} \mu_{\mathrm{s}}-N_{\mathrm{a}}^{a} \tilde{\mu}_{\mathrm{a}}+N_{\mathrm{a}}^{a} q_{\mathrm{a}} \Phi_{E}\right]-\frac{1}{2} c_{0}^{a}\left(\Phi_{0}^{a}-\Phi_{E}\right)^{2} \\
& :=\underbrace{\left[G_{\text {exc }, 0}^{a}+N_{\mathrm{a}}^{a}\left(q_{\mathrm{a}} \Phi_{E}-\tilde{\mu}_{\mathrm{a}}\right)\right]}_{G_{\text {exc, } \mathrm{CHE}}^{a}\left(\Phi_{E}\right)}-\underbrace{\frac{1}{2} c_{0}^{a}\left(\Phi_{0}^{a}-\Phi_{E}\right)^{2}}_{G_{\text {exc, DL }}^{a}\left(\Phi_{E}\right)} .
\end{aligned}
$$

The first bracket in Eq. (10) is identical to the excess free energy $G_{\text {exc, } \mathrm{CHE}}^{\alpha}\left(\Phi_{E}\right)$ as it would result within the $\mathrm{CHE}$ approach. The second term $G_{\text {exc,DL }}^{a}$ corresponds to the energy cost of charging the double layer.

This derivation highlights that the CHE approximation is equivalent to a first-order approximation in $\Phi_{E}$ of the FGC energetics. For each candidate structure $a$ the $\mathrm{CHE}$ energy expression is therefore accurate around the PZC of $a$ $\left(\Phi_{0}^{a}=\Phi_{E} \leftrightarrow N_{e}^{\text {net }}=0\right)$, where the term $G_{\text {exc,DL }}^{a}$ becomes small. On the other hand, for potentials much different than the PZC $\left(\Phi_{0}^{a} \neq \Phi_{E} \leftrightarrow N_{e}^{\text {net }} \neq 0\right)$ the additional energy contributions $G_{\text {exc,DL }}^{a}$ can become relevant. Within the CHE model, the only potentialdependence of $\mathcal{G}_{\text {exc }}^{a}\left(\Phi_{E}\right)$ arises from the term $N_{\mathrm{a}}^{a}\left(q_{\mathrm{a}} \Phi_{E}-\tilde{\mu}_{\mathrm{a}}\right)$. This dependence is thus always linear in $\Phi_{E}$, at variance with the typical parabolic potential dependence of electrochemical interface energies $^{5,47,68}$.

From the previous derivation we furthermore see that the relative stability of two electrode configurations $\alpha, \beta$ differing only in the number of adsorbates a and at applied potential $\Phi_{E}$ is

$\Delta \mathcal{G}_{\mathrm{exc}}^{a-\beta}\left(\Phi_{E}\right)=\Delta G_{\mathrm{exc}, \mathrm{CHE}}^{a-\beta}\left(\Phi_{E}\right)+\Delta G_{\mathrm{exc}, \mathrm{DL}}^{a-\beta}\left(\Phi_{E}\right)$,

where $\quad \Delta G_{\text {exc }, \text { CHE }}^{a-\beta}\left(\Phi_{E}\right)=G_{\text {exc }, 0}^{a}-G_{\text {exc }, 0}^{\beta}+\left(N_{\mathrm{a}}^{\alpha}-N_{\mathrm{a}}^{\beta}\right)\left(q_{\mathrm{a}} \Phi_{E}-\tilde{\mu}_{\mathrm{a}}\right)$

and $\Delta G_{\text {exc, DL }}^{a-\beta}\left(\Phi_{E}\right)=-\frac{1}{2} c_{0}^{a}\left(\Phi_{0}^{a}-\Phi_{E}\right)^{2}+\frac{1}{2} c_{0}^{\beta}\left(\Phi_{0}^{\beta}-\Phi_{E}\right)^{2}$.

Within the CHE approximation the relative stability of systems with the same number of adsorbates $N_{\mathrm{a}}^{\alpha}=N_{\mathrm{a}}^{\beta}$ is thus potentialindependent, excluding the possibility to simulate potentialdependent diffusion barriers, potential-induced shifts of stable adsorption sites, or potential-induced surface reconstructions that conserve the number of adsorbates (and generally also substrate atoms) at the electrode, as e.g., observed for the quasi hexagonal reconstruction of $\mathrm{Au}(100)^{5}$. Intriguingly, all these shortcomings are lifted when including the next higher order term in $\Phi_{E}$, namely the capacitive charging contribution of the double layer $G_{\text {exc.DL }}^{a-\beta}$-an energy contribution that is widespread in the classical electrochemical literature ${ }^{51-53,68-78}$. We will explicitly demonstrate below how this contribution leads to a shift of the electrosorption peaks with $\mathrm{pH}$, and how it relates to the so-called electrosorption valency, another classic concept in phenomenological electrochemistry ${ }^{51-53}$.

The double-layer correction $\Delta G_{\text {exc.DL }}^{a-\beta}\left(\Phi_{E}\right)$ can be further simplified by a change of variables according to $\Delta c_{0}=c_{0}^{a}-c_{0}^{\beta}$, 


$$
\begin{gathered}
\Phi_{0}=\frac{1}{2}\left(\Phi_{0}^{a}+\Phi_{0}^{\beta}\right), \text { and } \Delta \Phi_{0}=\Phi_{0}^{a}-\Phi_{0}^{\beta}, \text { which yields } \\
\Delta G_{\text {exc,DL }}^{a-\beta}\left(\Phi_{E}\right)=\underbrace{c_{0}^{\beta} \Delta \Phi_{0}}_{\Delta q^{\text {net }}}\left(\Phi_{E}-\Phi_{0}\right)- \\
-\frac{1}{2} \Delta c_{0}\left(\Phi_{E}-\left(\Phi_{0}+\frac{\Delta \Phi_{0}}{2}\right)\right)^{2} .
\end{gathered}
$$

In this notation, $\Delta G_{\text {exc,DL }}^{\alpha-\beta}$ becomes very suggestive. The first term corresponds to the (average) applied potential drop across the electric double layer $\left(\Phi_{E}-\bar{\Phi}_{0}\right)$ times the charge difference $\Delta q^{\text {net }}=c_{0}^{\beta} \Delta \Phi_{0}$ necessary to bring both systems $a$ and $\beta$ to an equal work function. We denote it with the superscript ${ }^{\text {net }}$, as the term corresponds to the difference in net surface charges (8) of both systems. As shall be shown below it can also be directly related to the adsorbate dipole ${ }^{79}$ and the electrosorption valency. The second term results from a change in the capacitive energy due to different interfacial capacitances $\Delta c_{0}$ of the two electrode configurations. Note that identical interface energy contributions were already proposed by Frumkin ${ }^{68}$ to understand electrosorption of charge-neutral species.

$\mathrm{pH}$ shifts of proton electrosorption peaks at platinum

Electrosorption peaks in cyclic voltammetry (CV) measurements are known to be sensitive probes of the electric double layer ${ }^{5,46,47}$. They therefore form ideal observables to assess critically the capabilities and quantitative performance of the CHE and the FGC approaches. Here, we concentrate in particular on proton electrosorption at two low-index single crystal surfaces of platinum, $\mathrm{Pt}(111)$ and $\mathrm{Pt}(100)$. For both systems, small but significant shifts of the electrosorption peaks with $\mathrm{pH}$ even on the RHE scale have been observed experimentally ${ }^{48-50}$. Such shifts go beyond what would simply be predicted by the Nernst equation and are certainly an intriguing object of study.

Within ab initio thermodynamics we approximate the electrosorption peak position ${ }^{5,46}$ as the electrode potential $U_{\mathrm{RHE}}^{\text {sorp }}$ on the reversible hydrogen electrode (RHE) scale for which the chargeequilibrated excess energy $\mathcal{G}_{\text {exc }}^{\mathrm{H}}$ of the hydrogen-covered electrode surface, with $N_{\mathrm{a}}^{\mathrm{H}}=N_{\mathrm{H}}$ specifically adsorbed $\mathrm{H}$ atoms, becomes equally stable as the charge-equilibrated excess energy $\mathcal{G}_{\text {exc }}^{\text {clean }}$ of the pristine, clean surface

$\Delta \mathcal{G}_{\text {exc }}^{\mathrm{H}-\text { clean }}\left(U_{\mathrm{RHE}}^{\text {sorp }}\right)=\mathcal{G}_{\text {exc }}^{\mathrm{H}}\left(U_{\mathrm{RHE}}^{\text {sorp }}\right)-\mathcal{G}_{\text {exc }}^{\text {clean }}\left(U_{\mathrm{RHE}}^{\text {sorp }}\right)=0$.

Electrode potentials on the absolute and on the RHE scale are hereby related as $\Phi_{E}=U_{\mathrm{RHE}}+4.44 \mathrm{~V}-\frac{k_{B} T}{\ell} \ln (10) \mathrm{pH}^{5,9,80}$. Inserting the second-order expansion of $\Delta \mathcal{G}_{\text {exc }}^{\mathrm{H}-\varepsilon_{\text {lean }}}$ (Eq. (11)) we thus arrive at the condition

$\Delta G_{\text {exc, CHE }}^{\mathrm{H}-\text { clean }}\left(U_{\mathrm{RHE}}^{\text {sorp,2nd }}\right)+\Delta G_{\text {exc,DL }}^{\mathrm{H}-\text { clean }}\left(U_{\mathrm{RHE}}^{\text {sorp,2nd }}\right)=0$,

where the first-order CHE term is

$$
\begin{aligned}
\Delta G_{\mathrm{exc}, \mathrm{CHE}}^{\mathrm{H}-\text { clean }}\left(U_{\mathrm{RHE}}^{\text {sorp }}\right)= & G_{\mathrm{exc}, 0}^{\mathrm{H}}-G_{\mathrm{exc}, 0}^{\text {clean }}+N_{\mathrm{H}}\left(e U_{\mathrm{RHE}}^{\text {sorp }}\right. \\
& \left.+4.44 \mathrm{eV}-k_{\mathrm{B}} T \ln (10) \mathrm{pH}-\tilde{\mu}_{\mathrm{H}}\right),
\end{aligned}
$$

and the double-layer correction is

$$
\begin{gathered}
\Delta G_{\text {exc }, \mathrm{DL}}^{\mathrm{H}-\text { clean }}\left(U_{\mathrm{RHE}}^{\text {sorp }}\right)=\Delta q^{\text {net }}\left(U_{\mathrm{RHE}}^{\text {sorp }}+\lambda_{1}(\mathrm{pH})\right)-\frac{1}{2} \Delta c_{0}\left(U_{\mathrm{RHE}}^{\text {sorp }}+\lambda_{2}(\mathrm{pH})\right)^{2}, \\
\text { where } \lambda_{1}(\mathrm{pH})=4.44 \mathrm{~V}-\bar{\Phi}_{0}-\frac{k_{\mathrm{B}} T}{e} \ln (10) \mathrm{pH} \\
\text { and } \lambda_{2}(\mathrm{pH})=\lambda_{1}(\mathrm{pH})-\frac{\Delta \Phi_{0}}{2} .
\end{gathered}
$$

Here, we have specified the general variables in Eqs. (11) and (14) for proton electrosorption as $q_{\mathrm{a}}=+e$ and $\tilde{\mu}_{\mathrm{a}}=\tilde{\mu}_{\mathrm{H}}$, and have introduced the charge difference between the clean and $\mathrm{H}$ covered surface $\Delta q^{\text {net }}=c_{0}^{\text {clean }} \Delta \Phi_{0}$, together with the extensive interfacial capacitance $c_{0}^{\text {clean }}=2 A C_{0}^{\text {clean }}$ of the clean surface, the difference in extensive interfacial capacitances $\Delta c_{0}=c_{0}^{\mathrm{H}}-c_{0}^{\text {clean }}$, and the difference $\Delta \Phi_{0}=\Phi_{0}^{\mathrm{H}}-\Phi_{0}^{\text {clean }}$ and average $\Phi_{0}=$
$1 / 2\left(\Phi_{0}^{\mathrm{H}}+\Phi_{0}^{\text {clean }}\right)$ of the work functions of the H-covered and clean surfaces, respectively.

Within the $\mathrm{CHE}$ approximation $\left(\Delta G_{\text {exc,DL }}^{\mathrm{H}-\text { clean }}=0\right)$, the condition is readily resolved for $U_{\mathrm{RHE}}^{\mathrm{SOrp}, \mathrm{CHE}}$ by exploiting the electrochemical potential of a proton, which is given by the SHE conditions $a s^{5}$

$\tilde{\mu}_{\mathrm{H}}=\frac{\mu\left(\mathrm{H}_{2}(g)\right)}{2}+4.44 \mathrm{eV}-k_{\mathrm{B}} T \ln (10) \mathrm{pH}$,

where $\mu\left(\mathrm{H}_{2}(g)\right)$ is the chemical potential of hydrogen gas at normal conditions ( $298 \mathrm{~K}, 1 \mathrm{bar}$ ). This then yields

$$
\begin{aligned}
U_{\mathrm{RHE}}^{\text {sorp }, \mathrm{CHE}}=- & -\frac{1}{e N_{\mathrm{H}}}\left(G_{\mathrm{exc}, 0}^{\mathrm{H}}-G_{\mathrm{exc}, 0}^{\text {clean }}-N_{\mathrm{H}} \frac{\mu\left(\mathrm{H}_{2}(g)\right)}{2}\right) \\
& \approx-\frac{1}{e}\left(\bar{E}_{\mathrm{ads}}^{\mathrm{H}}+\Delta F^{\mathrm{corr}, \mathrm{H}}\right),
\end{aligned}
$$

where in the second step we have followed the general approximation of Eq. (4) and introduced the average $\mathrm{H}$ adsorption energy $\bar{E}_{\text {ads }}^{H}$ plus the (negligibly small) free energy correction $\Delta F^{\mathrm{corr}, H}$ due to the change of vibrational and configurational degrees of freedom of the adsorbed hydrogen. The CHE approximation thus essentially predicts the proton electrosorption peak on the RHE scale simply at the potential corresponding to minus the average hydrogen adsorption energy. In fact, this holds in general, i.e., the CHE approximation would always equate electrosorption and adsorption for any kind of adsorbate. This is why in the CHE literature electrosorption and adsorption are often used interchangeably.

Most importantly, Eq. (20) does not depend anymore on $\mathrm{pH}$, i.e., within the $\mathrm{CHE}$ approximation the proton electrosorption peak does not exhibit any shifts on the RHE scale. If we were to transform $U_{\mathrm{RHE}}^{\text {sorp,CHE }}$ to an absolute scale, the peak would thus shift simply as predicted by the Nernst equation, at variance with the experimental observations (cf. further below). To this end, we acknowledge that lateral interactions among the adsorbed $\mathrm{H}$ atoms and configurational entropy terms might introduce a variation of the average adsorption energy and free energy correction term with surface coverage ${ }^{9}$. However, this will rather lead to a broadened electrosorption peak than natively introduce a pH-dependence in $U_{\mathrm{RHE}}^{\text {sorp, } \mathrm{CHE}}$. Similarly, different interface models in the underlying DFT calculations, e.g., the surface in vacuum, in various implicit solvation models or with differing adsorbed icelike water layers, will primarily affect only the absolute value of the calculated adsorption energy and therewith the predicted $\mathrm{pH}$ independent electrosorption peak position. The only way nonNernstian shifts can be rationalized within the CHE model is by a $\mathrm{pH}$-induced change in the adsorption energy, assuming e.g., a $\mathrm{pH}$ dependence of the interfacial water structure, or secondary effects such as the adsorption of co-ions, as further discussed below.

In contrast, already the second-order double-layer correction $\Delta G_{\text {exc } \mathrm{DL}}^{\mathrm{H}-\text { clean }}\left(U_{\mathrm{RHE}}^{\mathrm{sorp}}\right)$ introduces intrinsic $\mathrm{pH}$-dependencies, and leads to a quadratic equation defining $U_{\mathrm{RHE}}^{\text {sorp, 2nd }}$ (cf. Eqs. (16)-(18)). This quadratic equation and even more so the higher-order terms contained in the FGC approach yield therefore complex nonNernstian shifts of electrosorption peaks on the RHE scale. As an example, we point out that already the linear term in $\Delta G_{\text {exc,DL }}^{\mathrm{H}-\text { clean }}\left(U_{\mathrm{RHE}}^{\text {sorp }}\right)\left(\alpha \Delta q^{\text {net }}\right)$ effectively renormalizes the CHE peak position, while introducing an additional, linear dependence on $\mathrm{pH}$ as

$$
\begin{aligned}
U_{\mathrm{RHE}}^{\text {sorp, half }-2 \text { nd }}= & -\frac{\bar{E}_{\text {ads }}^{\mathrm{H}}+\Delta F^{\text {corr, }}+\Delta Q^{\text {net }}\left(4.44 \mathrm{~V}-\bar{\Phi}_{0}\right)}{e+\Delta Q^{\text {net }}} \\
& +\left(\frac{\Delta Q^{\text {net }}}{e+\Delta Q^{\text {net }}}\right) \frac{k_{B} T}{e} \ln (10) \mathrm{pH},
\end{aligned}
$$

where we have introduced the per-adsorbate quantity $\Delta Q^{\text {net }}=\frac{\Delta q^{\text {net }}}{N_{H}} . \Delta Q^{\text {net }}$ is largely independent of the surface coverage, given that the extensive quantity $\Delta q^{\text {net }}$ is expected to scale approximately linearly with $N_{\mathrm{H}}\left(\propto \Delta \Phi_{0}^{\alpha}\left(N_{\mathrm{H}}\right)\right.$, see e.g., Supplementary Fig. 10). Thus, whenever $\Delta Q^{\text {net }} / e$ is not vanishingly small, the corrections and linear $\mathrm{pH}$ shift of Eq. (21) become significant. 

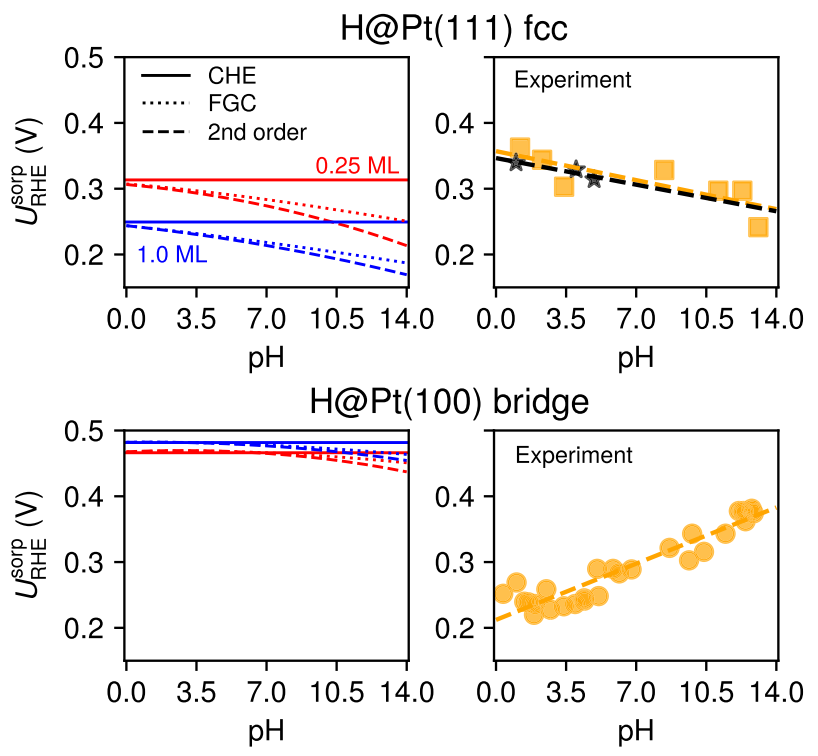

Fig. 1 Proton electrosorption potentials $U_{\mathrm{RHE}}^{\text {sorp }}$ for $\mathrm{Pt}(111)$ and $\mathrm{Pt}$ (100). Left panels: Calculated shifts within the CHE (solid line), the second-order (dashed line) and the FGC (dotted line) approach. Shown are results for $1 / 4 \mathrm{ML}$ (red) and $1 \mathrm{ML}$ (blue) hydrogen coverage on the surface. Right panels: Experimental shifts derived from works by Rizo et al. (orange squares) ${ }^{48}$ and Kamyabi et al. (black stars) $^{49}$ for Pt(111), as well as Zheng et al. (orange circles) ${ }^{50}$ for $\mathrm{Pt}(100)$. The linear fits are guide to the eye.

Table 1. Work function $\left(\Phi_{0}\right)$ and area-normalized interfacial capacitances $\left(C_{0}\right)$ at the PZC for the systems studied in the main text at the lowest (clean) and highest studied coverage $\theta$. More data in the SI.

\begin{tabular}{lllll}
\hline System & Adsorption site & $\begin{array}{l}\theta \\
(\mathrm{ML})\end{array}$ & $\begin{array}{l}\Phi_{0} \\
(\mathrm{~V})\end{array}$ & $\begin{array}{l}C_{0} \\
\left(\frac{\mu \mathrm{F}}{\mathrm{cm}^{2}}\right)\end{array}$ \\
\hline Clean Pt(100) & & - & 4.95 & 40 \\
Clean Pt(111) & & - & 4.97 & 36 \\
Clean $\mathrm{Ag}(111)$ & & - & 3.57 & 48 \\
$\mathrm{H} @ \mathrm{Pt}(100)$ & bridge & 1.00 & 5.02 & 22 \\
$\mathrm{H} @ \mathrm{Pt}(111)$ & $\mathrm{fcc}$ & 1.00 & 4.57 & 21 \\
$\mathrm{Cl} @ \mathrm{Ag}(111)$ & $\mathrm{fcc}$ & 0.33 & 4.32 & 34 \\
$\mathrm{Br} @ \mathrm{Ag}(111)$ & $\mathrm{fcc}$ & 0.33 & 4.30 & 32 \\
I@Ag(111) & $\mathrm{fcc}$ & 0.33 & 4.16 & 31 \\
\hline
\end{tabular}

Figure 1 illustrates the performance of the various approaches, by collecting the calculated proton electrosorption peak positions on $\mathrm{Pt}(111)$ and $\mathrm{Pt}(100)$. Concentrating first on the data obtained with an electrode surface model containing 1/4 ML hydrogen coverage in the energetically most stable fcc (Pt(111)) and bridge sites $(\mathrm{Pt}(100))$, respectively, we see qualitatively the same trends among the theoretical approaches on both surfaces. In contrast to the constant $U_{\mathrm{RHE}}^{\text {sorp, CHE }}$ predicted by the CHE approximation, already the second-order approximation yields non-Nernstian shifts of $U_{\mathrm{RHE}}^{\text {sorp,2nd }}$ at both surfaces. We note that a significant contribution to these shifts arises from the second, quadratic term in Eq. (18), i.e., from the large change in the interfacial capacitance $\Delta C_{0}$ upon proton electrosorption. In fact, for $\mathrm{Pt}(100)$, this contribution is large enough that it actually changes the shift direction from an upwards shift to higher $U_{\mathrm{RHE}}^{\text {sorp }}$ from the linear $\Delta q^{\text {net }}$ term only (cf. Eqs. (18) and (21) and the signs of the work function change for $\mathrm{H}$ on Pt in Table 1) to a small shift downwards. However, this holds, of course, only, if an implicit solvation model is used in the underlying DFT calculations as done in this work.
Using a surface slab in vacuum would yield highly underestimated differential interfacial capacitances and would correspondingly then also yield only minor shifts of the electrosorption potential even within the second-order approximation.

At both surfaces, the FGC approach yields shifts of the electrosorption potential $U_{\mathrm{RHE}}^{\text {sorp, } \mathrm{FGC}}$ that almost coincide with the second-order approximation at low $\mathrm{pH}$. Towards higher $\mathrm{pH}$, the two approaches then exhibit increasingly different results, with the FGC approach leading to smaller absolute shifts. These findings are consistently obtained also for other $\mathrm{H}$ adsorption sites and at other surface coverages (see Supplementary Figs. 5-9) . Figure 1 illustrates this explicitly by also showing the results calculated for full ML coverage. The primary effect of changing coverage is an offset of the various $U_{\mathrm{RHE}}^{\text {sorp }}$, as expected from the different average $\mathrm{H}$ adsorption energies. $U_{\mathrm{RHE}}^{\text {sorp,2nd }}$ and $U_{\mathrm{RHE}}^{\text {sorp, } F G C}$ exhibit only small changes of their slope and curvature. This is in agreement with the often rigid shift of experimental CV peaks at overall unaltered shape ${ }^{5}$. From the above derivations, we see that this will occur whenever the change of work function and interfacial capacitance scales approximately linearly with the surface coverage. Then the double-layer correction $G_{\text {exc,DL }}^{\mathrm{H}-\text { clean }}\left(U_{\mathrm{RHE}}^{\text {sorp }}\right)$ will scale similarly with $N_{\mathrm{H}}$ as the $\mathrm{CHE}$ term $G_{\mathrm{exc}, \mathrm{CHE}}^{\mathrm{H}-\text { clean }}\left(U_{\mathrm{RHE}}^{\text {sorp }}\right)$, and the $\mathrm{pH}$ terms in the overall condition for $U_{\mathrm{RHE}}^{\text {sorp,2nd }}$, Eq. (16), become coverage independent. Such a behavior can be expected for most adsorbates, whenever adsorbate-induced work function changes derive from local dipoles and capacitance variations from local alterations of the capacitance.

While the comparison of the various theoretical approaches sketches a rather consistent picture for both $\mathrm{Pt}(111)$ and $\mathrm{Pt}(100)$, clear differences arise in Fig. 1 in the comparison to the existing experimental data ${ }^{48-50}$. For $\mathrm{Pt}(111)$, the non-Nernstian shift of the proton electrosorption peak is reproduced very nicely by the FGC approach. Even though this holds also for the absolute positions, we note that this comparison is less well-defined. First, we infer only approximately the absolute experimental positions from the better resolved half peak-currents of the CVs in refs. ${ }^{48,49}$. (see also Supplementary Fig. 11). Second, absolute theoretical positions are sensitively affected by the employed approximate DFT exchangecorrelation functional, through the change in the $\mathrm{H}$ adsorption energy. The good agreement reached with the PBE functional could thus be partially fortuitous as discussed further below. More important is the fact that the FGC approach yields non-Nernstian shifts of the correct sign and order of magnitude without having to invoke a $\mathrm{pH}$-dependent change of the adsorption energy. To this end, it is important to point out that a previous theoretical $\mathrm{CHE}$ study by Karlberg et al. ${ }^{46}$ found no impact on the adsorption energy upon application of an electrostatic field and inclusion of explicit interfacial water molecules. In contrast, most recent results from ab initio molecular dynamics suggest a small influence of explicit water on average adsorption energies ${ }^{81}$. Nevertheless, even such refined dynamical simulations on neutral slabs, i.e., remaining at the CHE-level in terms of electrode charge equilibration, could not explain the non-Nernstian pH shifts of $U_{\mathrm{RHE}}^{\text {sorp }}$ at this surface. We therefore believe that the rationalization of these shifts within the FGC approach is indeed correct for the right reason, i.e., that the appropriate account of charge equilibration in the FGC scheme is key to describe proton electrosorption here.

On $\mathrm{Pt}(100)$ the performance of the FGC approach as measured by the existing experimental data is not as good. The peak position is significantly misaligned and the non-Nernstian shift with $\mathrm{pH}$ is predicted in the wrong direction and with wrong magnitude. The FGC approach predicts an almost negligible shift, in contrast to experiments where the magnitude of the shift is of comparable size as at $\mathrm{Pt}(111)$, just in the opposite direction. As already pointed out, the misalignment is most likely related to the 
exchange-correlation functional. A reevaluation of $U_{\mathrm{RHE}}^{\text {sorp, } \mathrm{CHE}}$ with the revPBE functional ${ }^{82,83}$ yields, for instance, a peak position lowered by $140 \mathrm{meV}$ to $0.33 \mathrm{~V}$, which agrees very well with the average experimental position. At the same time though, this functional would also lower the predicted $\mathrm{Pt}(111)$ peak position by $130 \mathrm{meV}$ and thus worsen the apparent agreement with experiment for this surface. Accepting a typical \pm 200 meV uncertainty of GGA-level absolute adsorption energies, the truly critical point assessing the description of the electrochemistry are therefore not the absolute positions, but the non-Nernstian shifts of the electrosorption peaks. To this end, the inability of our calculations to describe the experimental $\mathrm{pH}$ shifts at $\mathrm{Pt}(100)$ are most likely related to the absence of the explicit water structure in the present calculations. As shown convincingly in a recent study by Cheng et al. ${ }^{84}$, an increasing distance between metallic surface and interfacial water for higher $\mathrm{pH}$ values lead to a decrease in the adsorption energy and thereby to a peak shift of correct magnitude on the RHE scale. In general, any such influence of surface-specific interfacial water ${ }^{58,59,85-92}$ can not be captured in calculations based only on implicit solvation. This highlights that present-day FGC calculations still need to be seen as a numerically efficient approximation - that as a next step might need to be refined by appropriately extending them to mixed explicit/implicit solvation models ${ }^{39,58}$. We also note in passing that $\mathrm{Pt}(100)$ has no proper double layer region (co-adsorbed $\mathrm{H}, \mathrm{OH})^{49}$; co-ion effects might thus also contribute to the overall $\mathrm{pH}$-shift on the $\mathrm{RHE}$ scale $^{93}$. As such, proton electrosorption will remain an intriguing test system for future more explicit calculations to address the complexity of the interface.

\section{Electrosorption valencies of halide ions at $\mathrm{Ag}(111)$}

Electrosorption valencies are another fundamental and, as we shall see, closely related concept in the context of electrosorption in interfacial electrochemistry ${ }^{51,52}$. The electrosorption valency $l_{\mathrm{a}}$ (or formal charge number ${ }^{77}$ ) denotes the number of electrons that flows onto the electrode upon electrosorption of one adsorbate a at applied potential $\Phi_{E}$. While some debate exists of how to actually measure $I_{\mathrm{a}}$ or relate it to other concepts like partial charge transfer ${ }^{51-54,70-78}, l_{\mathrm{a}}$ is a well-defined thermodynamic quantity. According to the Lippmann equation ${ }^{47,65}$, the electronic surface charges are given by the derivative of the interface energy with respect to the applied potential. Within the present $a b$ initio thermodynamics framework, this definition translates to obtaining the absolute number of electrons $N_{e}^{a \text { abs }}\left(\Phi_{E}\right)$ of an electrode configuration $a$ from the derivative of the charge-equilibrated excess energy $\mathcal{G}_{\text {exc }}^{a}\left(\Phi_{E}\right)$ with respect to $\Phi_{E}$,

$N_{e}^{a, \mathrm{abs}}\left(\Phi_{E}\right)=\frac{1}{e} \frac{\partial \mathcal{G}_{\mathrm{exc}}^{a}\left(\Phi_{E}\right)}{\partial \Phi_{E}}$

Without loss of generality, we will focus here again on a symmetric slab setup, where on each of the two identical surfaces one adsorbate a electrosorbs to an empty site of an electrode configuration $a$ characterized by $N_{a}$ adsorbates of the same type and at the same site type. This results in a new electrode configuration with $2\left(N_{a}+1\right)$ adsorbates (and, as before, without changing the number of substrate atoms $N_{\mathrm{s}}$ ). The electrosorption valency of this process is then

$$
\begin{aligned}
l_{\mathrm{a}}\left(\Phi_{E}\right) & =\frac{1}{2}\left[N_{e}^{2\left(N_{\mathrm{a}}+1\right), \mathrm{abs}}\left(\Phi_{E}\right)-N_{e}^{2 N_{\mathrm{a}}, \mathrm{abs}}\left(\Phi_{E}\right)\right] \\
& =\frac{1}{2 e} \frac{\partial}{\partial \Phi_{E}} \Delta \mathcal{G}_{\mathrm{exc}}^{2\left(N_{\mathrm{a}}+1\right)-2 N_{\mathrm{a}}}\left(\Phi_{E}\right)
\end{aligned}
$$

where the factor $1 / 2$ renormalizes the symmetric slab setup. If we insert the general second-order expansion (Eqs. (11) and (14)), the electrosorption valency can again be separated into a CHE and a
DL part

$$
\begin{aligned}
& l_{\mathrm{a}}\left(\Phi_{E}\right)=l_{\mathrm{a}}^{\mathrm{CHE}}+I_{\mathrm{a}}^{\mathrm{DL}}\left(\Phi_{E}\right) \\
& =\frac{q_{\mathrm{a}}}{e}+\left[\frac{\Delta q^{\text {net }}}{2 e}-\frac{\Delta c_{0}^{2 N_{\mathrm{a}}}}{2 e}\left(\Phi_{E}-\Phi_{0}^{2\left(N_{\mathrm{a}}+1\right)}\right)\right] .
\end{aligned}
$$

At the CHE level we recover the assumption dictated by chargeneutrality that each adsorbed ion of charge $q_{\mathrm{a}}$ drags $q_{\mathrm{a}} / e$ electrons onto the surface. The electrosorption valency is thus purely given by the formal ionic charge: it is a constant integer number, independent of the applied potential and surface structure or composition. This simplification is lifted by the twoterm DL correction. The first potential-independent term renormalizes the formal ionic charge by the charge difference $\Delta q^{\text {net }}=$ $c_{0}^{2 N_{a}} \Delta \Phi_{0}$ and therewith allows for non-integer electrosorption valencies. The second term introduces an explicit potential dependence. To this end, it is important to point out that in this derivation both $\mathrm{DL}$ correction terms formally contain extensive differential capacitances. However, the intensive electrosorption valency is still well defined, as we consider the idealized situation where only one adsorbate electrosorbs on each side of the symmetric electrode slab.

This is further clarified when switching to a mean-field picture that characterizes the adsorbate layer only in terms of the surface coverage $\theta=N_{\mathrm{a}}{ }^{*} A_{\text {site }} / A$, where $A_{\text {site }}$ is the crystallographically determined surface area per adsorption site. This allows to describe the finite change in adsorbate numbers in terms of changing coverage, e.g.,

$$
\Delta q^{\text {net }}=2 A C_{0}^{2 N_{\mathrm{a}}}\left(\Phi_{0}^{2\left(N_{\mathrm{a}}+1\right)}-\Phi_{0}^{2 N_{\mathrm{a}}}\right) \approx 2 A C_{0}^{2 N_{\mathrm{a}}} \frac{\mathrm{d} \Phi_{0}^{N_{\mathrm{a}}}}{\mathrm{d} N_{\mathrm{a}}}=2 A_{\text {site }} C_{0}^{\theta} \frac{\mathrm{d} \Phi_{0}^{\theta}}{\mathrm{d} \theta} .
$$

This expression then contains only intensive quantities: $A_{\text {site, }}$ the differential capacitance $C_{0}^{\theta}$, and the derivative of the work function with respect to $\theta$. In this formulation, the DL-correction to the electrosorption valency becomes

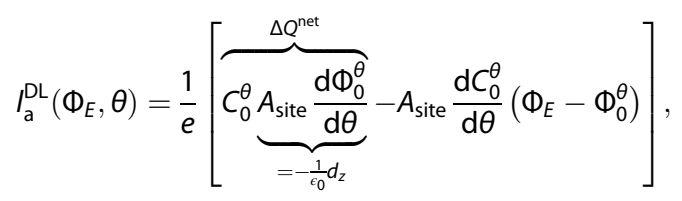

i.e., in essence a charge per adsorbate necessary to keep the work function constant upon electrosorption. $\Delta Q^{\text {net }}$ is identical to the term introduced before in the discussion of linear $\mathrm{pH}$ shifts for proton electrosorption in Eq. (21), where the derivative was approximated by the average change per adsorbate. Considering that $\frac{\mathrm{d} \Phi_{0}^{\theta}}{\mathrm{d} \theta}=-\frac{1}{\epsilon_{0} A_{\text {site }}} d_{z}$ can be related to the normal component of the adsorbate dipole $d_{z}{ }^{79}$ (with electrochemical sign convention), we recognize that the terms in Eq. (27) are closely related to the dipolar and capacitive contributions to the electrosorption valency that are recurrently discussed in classical electrochemistry works $47,51,52,94,95$. In fact, the relation between $d_{z}$ and $l_{a}$ is identically reported by Schmickler ${ }^{95}$.

To illustrate the effects of the DL corrections, we consider the electrosorption of halide ions $\left(\mathrm{Cl}^{-}, \mathrm{Br}^{-}, \mathrm{I}^{-}\right)$on $\mathrm{Ag}(111)$ in the lowcoverage regime. The experimentally well-established increase of their electrosorption valencies with increasing Pauling electronegativity ${ }^{53,54}$, i.e., $I_{1}^{\exp }<l_{\mathrm{Br}}^{\exp }<l_{\mathrm{Cl}}^{\exp }$, up to values that are only a fraction of the formal charge of -1 , poses a challenging test case. While the electrosorption valency is generally a function of applied potential and coverage (cf. Eq. (27) for the DL correction), we focus here on the $I_{\mathrm{a}}$ values in the coverage range up to $1 / 3 \mathrm{ML}$, at a given fixed potential. Specifically, we choose a potential value for which Foresti et al. observe ordered halide overlayers corresponding to $1 / 3 \mathrm{ML}^{54}$. After correcting for the underestimation of the PZC of $A g(111)$ with 

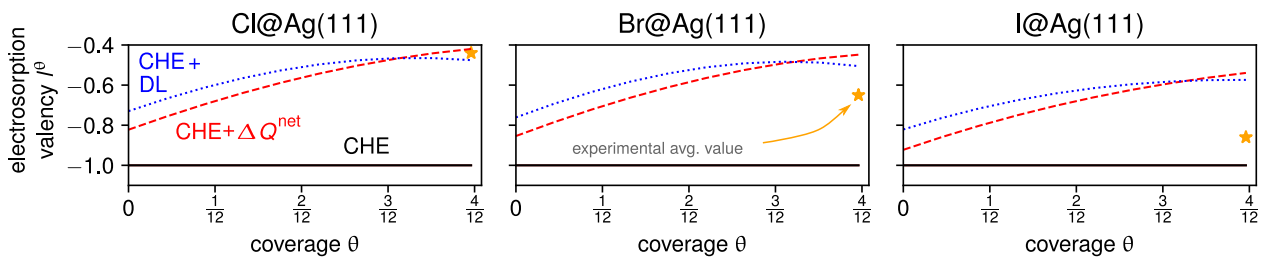

Fig. 2 Electrosorption valencies for halide ions in the low-coverage regime on the fcc sites of $A g(111)$ at a fixed potential $\left(\Phi_{E}(I)=4.0 \mathrm{~V}\right.$, $\Phi_{E}(\mathrm{Br})=4.1 \mathrm{~V}$ and $\Phi_{E}(\mathrm{Cl})=4.1 \mathrm{~V}$, see text). The valencies $\theta^{\theta}$ are calculated with the CHE approximation (solid black lines), the full secondorder double-layer (DL) correction (dotted blue lines) and the second-order $\Delta Q^{\text {net }}$ term (dashed red lines) (see Eqs. (25) and (27)). Experimental "integral" electrosorption valencies for the low-coverage regime are from Foresti et al. ${ }^{54}$ (orange stars).

the current solvent parameters $(-0.42 \mathrm{eV})$, we have: $\Phi_{E}(\mathrm{l})=4.0 \mathrm{~V}$, $\Phi_{E}(\mathrm{Br})=4.1 \mathrm{~V}$ and $\Phi_{E}(\mathrm{Cl})=4.1 \mathrm{~V}$. Interpolation of $\Phi_{0}^{\theta}$ and $C_{0}^{\theta}$ computed at coverages between $1 / 12$ an $1 / 3 \mathrm{ML}$ (see Supplementary Figs. 12 and 13) allows to determine the derivatives in Eq. (27) and therefore the double-layer correction to the electrosorption valencies. Figure 2 provides the corresponding valencies, computed with the $\mathrm{CHE}$ approximation, with the full second-order double-layer correction and only considering the second-order $\Delta Q^{\text {net }}$ term. By construction, the CHE approximation yields the formal charge -1 for the entire halide ion series. Instead, the second-order terms introduce varying non-integer values which vary with coverage. To this end, it is important to note that the extrapolations of the corresponding lines to the limit $\theta \rightarrow 0$ do not coincide with the CHE results, supporting the fundamental differences between FGC and CHE calculations also for individual adsorbates in the limit of infinite cell size. In this respect, it is reassuring to see that already the consideration of the second-order $\Delta Q^{\text {net }}$ term reproduces the expected trend of the computed electrosorption valencies with the Pauling electronegativity over the entire low-coverage range. This also extends to the full DL correction, but the actual values obtained depend then on the chosen applied potential (cf. Eq. (27)). Deferring a detailed comparison to experimental data to a forthcoming publication, we include in Fig. 2 measured "integral" electrosorption valencies for low coverages ${ }^{54}$. Also in light of the large uncertainties of different measurement methods ${ }^{53,54}$, the agreement obtained with the DL corrected calculations is very encouraging, and confirms the importance of FGC approaches toward a reliable first-principles description of electrified interfaces.

\section{DISCUSSION}

Ab initio thermodynamics offers a computationally efficient and ideally predictive access to questions in interfacial electrochemistry. In the CHE approximation it enjoys high popularity, in particular in applications to electrocatalysis. In this work, we used electrosorption as a fundamental elementary step in electrocatalysis to compare this approach to FGC ab initio thermodynamics. The present analysis underscores the importance of double-layer charging, missing in the zero net-charge CHE approach. In the context of electrosorption this manifests itself in the ability to describe non-Nernstian $\mathrm{pH}$-shifts of electrosorption peaks and non-integer electrosorption valencies; both effects can be captured at the variable-charge FGC level. As evaluated against existing experimental data for proton electrosorption at $\mathrm{Pt}$ electrodes, and halide ion electrosorption at Ag electrodes, the present first-principles description yields in general results closer to experiment than pure CHE calculations and is partly already semi-quantitative. Analysis of a second-order approximation to the FGC energetics allows to rationalize the relevance of the potential of zero charge, the interfacial capacitance, and work function shifts induced by adsorbates e.g., through their dipoles. All the expressions derived here for the electrochemical stability of adsorbates and their electrosorption valencies are in agreement with classical electrochemistry works ${ }^{47,51-53,68-78,94,95}$, closing the gap between phenomenological and first-principles treatments of electrified interfaces.

On a more practical level, the analysis with the second-order model provides clear insight into the limitations of zero-charge calculations and guidelines for their usage:

- Increased deviations of the CHE energetics from FGC results are expected whenever the electrode potentials $\Phi_{E}$ considered are significantly different from the potential of zero charge $\Phi_{0}$, as the correction terms scale with $\left(\Phi_{E}-\Phi_{0}\right)$.

- Energetic differences between CHE and FGC calculations are proportional to the magnitude of the interfacial capacitance $C_{0}$; CHE energetics thus becomes problematic for high electrolyte concentrations with large $C_{0}$ values. Furthermore, a non-negligible influence of the electrolyte chemistry can be expected, due to ion-specific interfacial capacitances ${ }^{96,97}$. DFT slab calculations in vacuum underestimate $C_{0}$ significantly and do therefore not allow to assess the effects of an applied potential.

- Major differences between CHE and FGC calculations will occur for adsorbates with significant dipole moments, whenever the work function change per adsorbate is large. This is in line with other studies ${ }^{30,40}$ and was noted early ${ }^{7,98}$.

The improved description at the FGC level comes at a somewhat increased computational cost. This does not so much refer to the actual DFT calculations themselves, thanks to efficient implementations of current implicit solvation models. Instead, it refers to the larger number of DFT calculations of the system in different charge states. Depending on the specific application this may typically mean $\sim 10$ times more calculations. While this will typically not be prohibitive, we note that the number of calculations required to determine the quantities for the secondorder model is generally smaller, as it only includes parameters obtained at the PZC, namely adsorption energies, $\Phi_{0}^{a}$ and the interfacial capacitance $C_{0}^{a}$. Considering the success of this model in describing the electrosorption phenomena studied in this work, calculations at this level might therefore be an appealing intermediate option for systems where computational cost is truly limiting. Having said this, we emphasize that the remaining inaccuracies of FGC calculations can still be significant. Notable factors are the approximate DFT exchange-correlation functional and their errors in adsorption energies, the neglect of explicit water $^{58,59,86}$, or missing co-ions ${ }^{93}$. In addition, the present analytical derivations indicate that discrepancies between allimplicit and all-explicit interfacial capacitances $39,55,92,99$ and work functions ${ }^{58}$ can have an impact as well. We expect many of these limitations to be overcome by FGC-type schemes with implicit/ explicit hybrid descriptions of interfacial water, an approach we will pursue in the future.

\section{METHODS}

DFT calculations

All DFT calculations reported below are performed with the Quantum ESPRESSO package ${ }^{100}$ (PWscf), the PBE exchange-correlation functional ${ }^{101}$ 
a) DFT energy

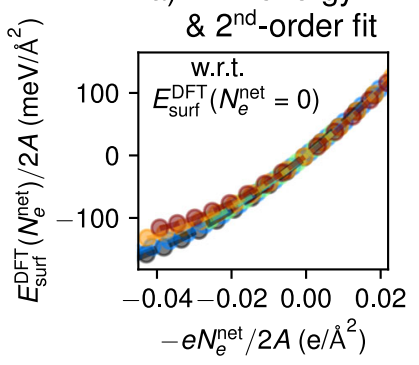

c) charge-equilibrated excess energies and $2^{\text {nd }}$-order fit

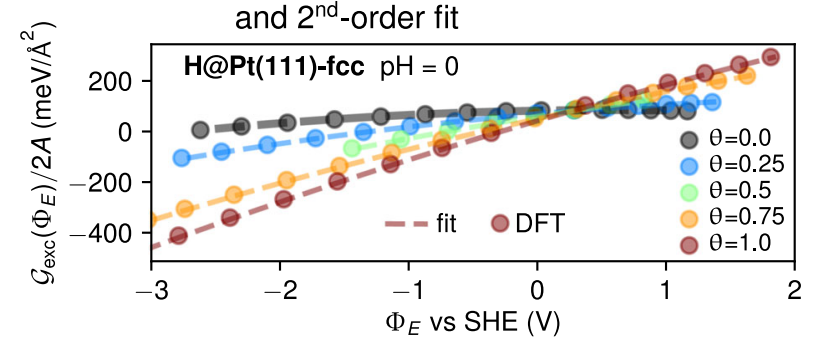

Fig. 3 Area-normalized interface energetics of pristine and $\mathrm{H}(\mathrm{fcc})$ covered $\mathrm{Pt}(111)$ as obtained within the SCCS implicit solvation model. Dots are the DFT calculated values, lines are polynomial interpolations. a Calculated total energy vs. system charge $N_{e}^{\text {net }}$ including a second-order polynomial fit. $\mathbf{b}$ Fit residues for a secondand fifth-order fit. c Charge-equilibrated excess energies $\mathcal{G}_{\text {exc }}^{a}\left(\Phi_{E}\right) / 2 A$ for clean and $\mathrm{H}(\mathrm{fcc})$-covered $\mathrm{Pt}(111)$ at applied electrode potential $\Phi_{E}$ for $\mathrm{pH}=0$, including second-order fits.

and pseudopotentials from the SSSP library ${ }^{102}$ (v0.7, PBE, efficiency) with density and wave function cutoffs of 360 and 45 Ry, respectively. The two systems, $\mathrm{H}$ on $\mathrm{Pt}$ and halide ions on $\mathrm{Ag}$ are studied in a periodic, symmetric slab setup with substrate and adsorbate degrees of freedom described explicitly. For Pt we use $(2 \times 2)$ cells with 7 and 8 Pt layers for the $(111)$ and (100) surfaces, respectively; for $\mathrm{Ag}(111)$ we use a $(\sqrt{12} \times \sqrt{12})$ supercell consisting of $6 \mathrm{Ag}$ layers. Slabs are separated by $\approx 17 \AA$. Brillouin zone integrations are performed using $\Gamma$-centered Monkhorst-Pack meshes (Pt: $(10 \times 10 \times 1), \mathrm{Ag:}(4 \times 4 \times 1))$ and a cold smearing ${ }^{103}$ of $0.01 \mathrm{Ry}(\mathrm{Pt})$ or 0.02 Ry (Ag), yielding a numerical accuracy better than $1 \mathrm{meV} / \AA^{2}$ in the interface energies.

As implicit solvation model we use the SCCS implementation of ENVIRON ${ }^{18,36,104,105}$ with optimized interfacial parameters $\left(\rho_{\min }=0.0013\right.$, $\rho_{\max }=0.01025, a=\beta=\gamma=0$ ) and a Helmholtz-layer representation of the electrolyte via planar counter charges. For this setup, good agreement is found between experimentally measured and predicted PZCs, interfacial capacitances, and electrosorption behavior for Pt electrodes, as reported in earlier work ${ }^{5}$. In particular, the work function-when determined as the energy difference between the Fermi level and the flat electrostatic potential in the implicit region-and the electrode potential $\Phi_{E}$ on the absolute scale are identical, which is why all electrochemical potentials (ionic and electronic) in this work are expressed on the absolute scale.

As reference energies for the adsorbates we use the Gibbs free energies of the diatomic molecules ( $p=1 \mathrm{bar}, T=298 \mathrm{~K}$ ) in vacuum including the vibrational and rotational degrees of freedom. The free energy corrections $\Delta F^{\text {corr }}$ are determined as the Helmholtz free energy contributions due to coverage-independent vibrations of the adsorbed species (calculated via finite-differences), neglecting small configurational entropy contributions. Specifically, in the case of H@Pt we thus discriminate only between hollow and bridge sites with the (111)-fcc and (100)-bridge vibrations for 0.25 monolayer (ML) coverage taken as per-adsorbate values.

For the determination of the charge-equilibrated excess energies, we optimize the geometries for the interfacial candidate structures for a set of finite surface charges $N_{e}^{\text {net }}(\geq 9$ values centered around 0 ) and evaluate their work functions $\Phi^{a}$ and total energies $E_{\text {surf }}^{\mathrm{DFT}, a}$, as exemplified in Fig. 3a for the case of $\mathrm{H}$ at fcc sites on $\mathrm{Pt}(111)$. Values are chosen to sample a potential range of $\sim 4-5 \mathrm{~V}$ around the PZC $\left(N_{e}^{\text {net }}=2 A C_{0}\left(\Phi_{E}-\Phi_{0}\right)\right.$ with $C_{0} \approx 30 \mu \mathrm{F} /$ $\mathrm{cm}^{2}$ ) for $\mathrm{H}$ on $\mathrm{Pt}$, and in a smaller range of $\sim 2 \mathrm{~V}$ for the halide systems. As described above and more thoroughly in ref. ${ }^{5}$, charge-equilibrated excess energies $\mathcal{G}_{\text {exc }}^{a}$ (dots in Fig. 3c) can then be obtained directly for the set of electrode potentials $\Phi_{E}$, which correspond to the obtained set of work functions $\Phi^{\alpha}\left(N_{e}^{\text {net }, \alpha}\right)$. This involves evaluation of Eq. (1) with the described approximations and for the DFT energies with corresponding surface charges $E_{\text {surf }}^{\mathrm{DFT}, \alpha}\left(N_{e}^{\text {net, } \alpha}\left(\Phi^{\alpha}\right)\right)$ (dots in Fig. 3a). The lines in Fig. 3 correspond to polynomial interpolations using the data of the studied set of charges. The close agreement between a second-order model and the FGC results for this system are supported by the reported residuals in Fig. $3 \mathrm{~b}$, and the accurate parabolic interpolation in Fig. $3 c$ (error $\sim 1 \mathrm{meV} / \AA^{2}$ ) (see also Supplementary Figs. 1-4). The interfacial capacitances follow directly from the second derivative of the interpolant (cf. Eq. (9)). Table 1 reports work functions $\Phi_{0}$ and area-normalized interfacial capacitances $C_{0}$ for the systems studied in the main text, at the lowest and highest coverage. Values for other coverages and adsorption sites are reported in the Supplementary Tables 1-4.

\section{DATA AVAILABILITY}

All relevant computational results are provided in the reported tables.

\section{CODE AVAILABILITY}

All results were obtained with the open source code Quantum ESPRESSO. Postprocessing scripts can be provided on request from the corresponding author.

Received: 6 May 2020; Accepted: 24 July 2020;

Published online: 08 September 2020

\section{REFERENCES}

1. Lozovoi, A. Y., Alavi, A., Kohanoff, J. \& Lynden-Bell, R. M. Ab initio simulation of charged slabs at constant chemical potential. J. Chem. Phys. 115, 1661-1669 (2001).

2. Nørskov, J. K. et al. Origin of the overpotential for oxygen reduction at a fuel-cell cathode. J. Phys. Chem. B 108, 17886-17892 (2004).

3. Rogal, J. \& Reuter, K. Ab initio atomistic thermodynamics for surfaces: a primer. in Experiment, Modeling and Simulation of Gas- Surface Interactions for Reactive Flows in Hypersonic Flights. pp. 2-1-2-18. Educational Notes RTO-EN-AVT-142, Paper 2. Neuilly-sur-Seine (2007).

4. Reuter, K. Ab initio thermodynamics and first-principles microkinetics for surface catalysis. Catal. Lett. 146, 541-563 (2016).

5. Hörmann, N. G., Andreussi, O. \& Marzari, N. Grand canonical simulations of electrochemical interfaces in implicit solvation models. J. Chem. Phys. 150, 041730 (2019).

6. Nørskov, J. et al. Trends in the exchange current for hydrogen evolution. J. Electrochem. Soc. 152, J23-J26 (2005).

7. Rossmeisl, J., Nørskov, J. K., Taylor, C. D., Janik, M. J. \& Neurock, M. Calculated phase diagrams for the electrochemical oxidation and reduction of water over Pt(111). J. Phys. Chem. B 110, 21833-21839 (2006).

8. Fang, Y.-H. \& Liu, Z.-P. Mechanism and tafel lines of electro-oxidation of water to oxygen on RuO2(110). J. Am. Chem. Soc. 132, 18214-18222 (2010).

9. Bonnet, N. \& Marzari, N. First-principles prediction of the equilibrium shape of nanoparticles under realistic electrochemical conditions. Phys. Rev. Lett. 110, 086104 (2013).

10. Calle-Vallejo, F. \& Koper, M. T. First-principles computational electrochemistry: achievements and challenges. Electrochim. Acta 84, 3-11 (2012).

11. Huang, J. et al. Potential-induced nanoclustering of metallic catalysts during electrochemical CO2 reduction. Nat. Commun. 9, 3117 (2018).

12. Peterson, A. A., Abild-Pedersen, F., Studt, F., Rossmeisl, J. \& Nørskov, J. K. How copper catalyzes the electroreduction of carbon dioxide into hydrocarbon fuels. Energy Environ. Sci. 3, 1311-1315 (2010).

13. Lozovoi, A. Y. \& Alavi, A. Reconstruction of charged surfaces: general trends and a case study of Pt(110) and Au(110). Phys. Rev. B 68, 245416 (2003).

14. Otani, M. \& Sugino, O. First-principles calculations of charged surfaces and interfaces: a plane-wave nonrepeated slab approach. Phys. Rev. B 73, 115407 (2006).

15. Dabo, I., Cancès, E., Li, Y. L. \& Marzari, N. Towards First-Principles Electrochemistry. Preprint at https://arxiv.org/abs/0901.0096 (2008).

16. Dabo, I., Li, Y., Bonnet, N. \& Marzari, N. Ab Initio Electrochemical Properties of Electrode Surfaces. Chapter 13, 415-431 (Wiley-Blackwell, 2010).

17. Jinnouchi, R. \& Anderson, A. B. Electronic structure calculations of liquid-solid interfaces: Combination of density functional theory and modified PoissonBoltzmann theory. Phys. Rev. B 77, 245417 (2008).

18. Andreussi, O., Dabo, I. \& Marzari, N. Revised self-consistent continuum solvation in electronic-structure calculations. J. Chem. Phys. 136, 064102 (2012). 
19. Letchworth-Weaver, K. \& Arias, T. A. Joint density functional theory of the electrode-electrolyte interface: Application to fixed electrode potentials, interfacial capacitances, and potentials of zero charge. Phys. Rev. B 86, 075140 (2012).

20. Bonnet, N., Dabo, I. \& Marzari, N. Chemisorbed molecules under potential bias: detailed insights from first-principles vibrational spectroscopies. Electrochim. Acta 121, 210-214 (2014).

21. Mathew, K., Sundararaman, R., Letchworth-Weaver, K., Arias, T. A. \& Hennig, R. G. Implicit solvation model for density-functional study of nanocrystal surfaces and reaction pathways. J. Chem. Phys. 140, 084106 (2014).

22. Lespes, N. \& Filhol, J.-S. Using implicit solvent in ab initio electrochemical modeling: investigating $\mathrm{Li}+/ \mathrm{Li}$ electrochemistry at a $\mathrm{Li} /$ solvent interface. J. Chem. Theory Comput. 11, 3375-3382 (2015).

23. Fisicaro, G., Genovese, L., Andreussi, O., Marzari, N. \& Goedecker, S. A generalized Poisson and Poisson-Boltzmann solver for electrostatic environments. J. Chem. Phys. 144, 014103 (2016).

24. Fisicaro, G. et al. Soft-sphere continuum solvation in electronic-structure calculations. J. Chem. Theory Comput. 13, 3829-3845 (2017).

25. Ringe, S., Oberhofer, H. \& Reuter, K. Transferable ionic parameters for firstprinciples Poisson-Boltzmann solvation calculations: neutral solutes in aqueous monovalent salt solutions. J. Chem. Phys. 146, 134103 (2017).

26. Sundararaman, R. \& Schwarz, K. Evaluating continuum solvation models for the electrode-electrolyte interface: challenges and strategies for improvement. J. Chem. Phys. 146, 084111 (2017).

27. Sundararaman, R., Goddard, W. A. \& Arias, T. A. Grand canonical electronic density-functional theory: algorithms and applications to electrochemistry. J. Chem. Phys. 146, 114104 (2017).

28. Sundararaman, R., Figueiredo, M. C., Koper, M. T. M. \& Schwarz, K. A. Electrochemical capacitance of CO-terminated $\mathrm{Pt}(111)$ dominated by the CO-solvent gap. J. Phys. Chem. Lett. 8, 5344-5348 (2017).

29. Ping, Y., Nielsen, R. J. \& Goddard, W. A. The reaction mechanism with free energy barriers at constant potentials for the oxygen evolution reaction at the IrO2 (110) surface. J. Am. Chem. Soc. 139, 149-155 (2017).

30. Zhang, H., Goddard, W. A., Lu, Q. \& Cheng, M.-J. The importance of grandcanonical quantum mechanical methods to describe the effect of electrode potential on the stability of intermediates involved in both electrochemical $\mathrm{CO} 2$ reduction and hydrogen evolution. Phys. Chem. Chem. Phys. 20, 2549-2557 (2018).

31. Kastlunger, G., Lindgren, P. \& Peterson, A. A. Controlled-potential simulation of elementary electrochemical reactions: proton discharge on metal surfaces. $J$. Phys. Chem. C 122, 12771-12781 (2018).

32. Nattino, F., Truscott, M., Marzari, N. \& Andreussi, O. Continuum models of the electrochemical diffuse layer in electronic-structure calculations. J. Chem. Phys. 150, 041722 (2019).

33. Andreussi, $O$. et al. Solvent-aware interfaces in continuum solvation. J. Chem. Theory Comput. 15, 1996-2009 (2019).

34. Gauthier, J. A. et al. Challenges in modeling electrochemical reaction energetics with polarizable continuum models. ACS Catal. 9, 920-931 (2019).

35. Andreussi, O., Nattino, F. \& Hörmann, N. G. Chapter: Continuum Embedding Models for Electrolyte Solutions in First-Principles Simulations of Electrochemistry. in (eds Melander, M., Laurila, T. \& Laasonen, K.) Atomic-Scale Modelling of Electrochemical Systems. (John Wiley and Sons Ltd., 2020) (in press).

36. ENVIRON package. http://www.quantum-environment.org.

37. Held, A. \& Walter, M. Simplified continuum solvent model with a smooth cavity based on volumetric data. J. Chem. Phys. 141, 174108 (2014).

38. Sundararaman, R. et al. JDFTx: software for joint density-functional theory SoftwareX 6, 278-284 (2017).

39. Gauthier, J. A. et al. Unified approach to implicit and explicit solvent simulations of electrochemical reaction energetics. J. Chem. Theory Comput. 15, 6895-6906 (2019).

40. Ringe, $\mathrm{S}$. et al. Double layer charging driven carbon dioxide adsorption limits the rate of electrochemical carbon dioxide reduction on Gold. Nat. Commun. 11, 33 (2020).

41. Venkatachalam, S., Kaghazchi, P., Kibler, L. A., Kolb, D. M. \& Jacob, T. First principles studies of the potential-induced lifting of the $A u(100)$ surface reconstruction. Chem. Phys. Lett. 455, 47-51 (2008).

42. Mamatkulov, M. \& Filhol, J.-S. An abinitio study of electrochemical vs. electromechanical properties: the case of $\mathrm{CO}$ adsorbed on a $\mathrm{Pt}(111)$ surface. Phys. Chem. Chem. Phys. 13, 7675-7684 (2011).

43. Filhol, J.-S. \& Doublet, M.-L. An ab initio study of surface electrochemical disproportionation: the case of a water monolayer adsorbed on a $\mathrm{Pd}(111)$ surface. Catal. Today 202, 87-97 (2013).

44. Steinmann, S. N., Michel, C., Schwiedernoch, R. \& Sautet, P. Impacts of electrode potentials and solvents on the electroreduction of $\mathrm{CO} 2$ : a comparison of theoretical approaches. Phys. Chem. Chem. Phys. 17, 13949-13963 (2015).

45. Weitzner, S. E. \& Dabo, I. Quantum-continuum simulation of underpotentia deposition at electrified metal-solution interfaces. npj Comput. Mater. 3, 1 (2017).
46. Karlberg, G. S. et al. Cyclic voltammograms for $\mathrm{H}$ on $\mathrm{Pt}(111)$ and $\mathrm{Pt}(100)$ from first principles. Phys. Rev. Lett. 99, 126101 (2007).

47. Schmickler, W. \& Santos, E. Interfacial Electrochemistry. 2nd edn. (Springer Verlag Berlin Heidelberg, Berlin, Heidelberg, 2010).

48. Rizo, R., Sitta, E., Herrero, E., Climent, V. \& Feliu, J. M. Towards the understanding of the interfacial pH scale at $\mathrm{Pt}\left(\begin{array}{lll}1 & 1 & 1\end{array}\right)$ electrodes. Electrochim. Acta 162, 138-145 (2015).

49. Kamyabi, M. A., Martínez-Hincapié, R., Feliu, J. M. \& Herrero, E. Effects of the interfacial structure on the methanol oxidation on platinum single crystal electrodes. Surfaces 2, 177-192 (2019).

50. Zheng, J., Sheng, W., Zhuang, Z., Xu, B. \& Yan, Y. Universal dependence of hydrogen oxidation and evolution reaction activity of platinum-group metals on $\mathrm{pH}$ and hydrogen binding energy. Sci. Adv. 2, e1501602 (2016).

51. Vetter, K. J. \& Schultze, J. W. Potentialabhängigkeit von elektrosorptionsgleichgewichten und die elektrosorptionswertigkeit $\gamma$. Ber. Bunsenges. Phys. Chem. 76, 920-927 (1972).

52. Vetter, K. J. \& Schultze, J. W. Stromfluc bei elektrosorptionsprozessen und elektrosorptionswertigkeit $\gamma$. Ber. Bunsenges. Phys. Chem. 76, 927-933 (1972).

53. Guidelli, R. \& Schmickler, W. Electrosorption Valency and Partial Charge Transfer, 303-371 (Springer, US, Boston, MA, 2005).

54. Foresti, M. L., Innocenti, M., Forni, F. \& Guidelli, R. Electrosorption valency and partial charge transfer in halide and sulfide adsorption on $\mathrm{Ag}(111)$. Langmuir 14 7008-7016 (1998)

55. Schmickler, W., Double layer theory. J. Solid State Electrochem. (2020).

56. Trasatti, S. The "absolute" electrode potential-the end of the story. Electrochim. Acta 35, 269-271 (1990).

57. Zhu, B., Xu, Z., Wang, C. \& Gao, Y. Shape evolution of metal nanoparticles in water vapor environment. Nano Lett. 16, 2628-2632 (2016).

58. Hörmann, N. G. et al. Absolute band alignment at semiconductor-water interfaces using explicit and implicit descriptions for liquid water. npj Comput. Mater. 5, 100 (2019).

59. Heenen, H. H., Gauthier, J. A., Kristoffersen, H. H., Ludwig, T. \& Chan, K. Solvation at metal/water interfaces: An ab initio molecular dynamics benchmark of common computational approaches. J. Chem. Phys. 152, 144703 (2020).

60. Hörmann, N. \& Groß, A. Stability, composition and properties of $\mathrm{Li}_{2} \mathrm{FeSiO}_{4}$ surfaces studied by DFT. J. Solid State Electrochem. 18, 1401-1413 (2014).

61. Hörmann, N. et al. Some challenges in the first-principles modeling of structures and processes in electrochemical energy storage and transfer. J. Power Sources 275, 531-538 (2015).

62. Rossmeisl, J., Chan, K., Ahmed, R., Tripkovic, V. \& Björketun, M. E. pH in atomic scale simulations of electrochemical interfaces. Phys. Chem. Chem. Phys. 15, 10321-10325 (2013).

63. Filhol, J.-S. \& Neurock, M. Elucidation of the electrochemical activation of water over Pd by first principles. Angew. Chem. Int. Ed. 45, 402-406 (2006).

64. Skúlason, E. et al. Density functional theory calculations for the hydrogen evolution reaction in an electrochemical double layer on the $\mathrm{Pt}(111)$ electrode. Phys. Chem. Chem. Phys. 9, 3241-3250 (2007).

65. Lippmann, G. Relations entre les phenomènes électriques et capillaires. Ann. Chim. Phys. 5, 494 (1875).

66. Badiali, J. P. \& Goodisman, J. Lippmann equation and the ideally polarizable electrode. J. Phys. Chem. 79, 223-232 (1975).

67. Mugele, F. \& Baret, J.-C. Electrowetting: from basics to applications. J. Phys. 17, R705-R774 (2005).

68. Frumkin, A. Über die beeinflussung der adsorption von neutralmolekülen durch ein elektrisches feld. Z. Phys. 35, 792-802 (1926).

69. Butler, J. A. V. \& Walker, J. The equilibrium of heterogeneous systems including electrolytes. Part III. The effect of an electric field on the adsorption of organic molecules, and the interpretation of electro-capillary curves. Proc. Roy. Soc. A 122, 399-416 (1929).

70. Lorenz, W. \& Salié, G. Zum Mechanismus der elektrochemischen phasengrenzreaktion. Z. Phys. Chem. 218, 259 (1962).

71. Schultze, J. \& Vetter, K. Experimental determination and interpretation of the electrosorption valency $\gamma$. J. Electroanal. Chem. Interf. Electrochem. 44, 63-81 (1973).

72. Frumkin, A., Damaskin, B. \& Petrii, O. On the charge transfer in the process of adsorption at the electrode/solution interface: remarks on the paper experimental determination and interpretation of the electrosorption valency $\gamma$ by $\mathrm{J}$. Schultze and K. Vetter. J. Electroanal. Chem. Interf. Electrochem. 53, 57-65 (1974).

73. Vetter, K. \& Schultze, J. General aspects of the electrosorption valency thermodynamic problems and nonthermodynamic analysis: discussion of the paper by A. Frumkin, B. Damaskin and O. Petrii. J. Electroanal. Chem. Interf. Electrochem. 53, 67-76 (1974).

74. Lorenz, W. \& Salié, G. Partial charge transfer reactions in electrochemical kinetics: review on the theory of measuring methods for electrode processes with adsorbed intermediates. J. Electroanal. Chem. Interf. Electrochem. 80, 1-56 (1977). 
75. Valette, G., Hamelin, A. \& Roger, P. Specific adsorption on silver single crystals in aqueous solutions. Z. Phys. Chem. 113, 71 (1978).

76. Habib, M. \& Bockris, J. Specific Adsorption of lons. Vol. 1. 135-219 (Springer, Boston, MA, 1980).

77. Trasatti, S. \& Parsons, R. Interphases in systems of conducting phases (Provisional). Pure Appl. Chem. 55, 1251 (1983).

78. de Levie, R. The electrosorption valency and partial charge transfer. J. Electroanal. Chem. 562, 273-276 (2004).

79. Gossenberger, F., Roman, T., Forster-Tonigold, K. \& Groß, A. Change of the work function of platinum electrodes induced by halide adsorption. Beilstein $J$. Nanotechnol. 5, 152-161 (2014).

80. Trasatti, S. Systematic trends in the crystal face specificity of interfacial parameters: the cases of Ag and Au. J. Electroanal. Chem. 329, 237-246 (1992).

81. Yang, G. et al. The nature of hydrogen adsorption on platinum in the aqueous phase. Angew. Chem. Int. Ed. 58, 3527-3532 (2019).

82. Zhang, Y. \& Yang, W. Comment on "Generalized Gradient Approximation Made Simple". Phys. Rev. Lett. 80, 890-890 (1998).

83. Hammer, B., Hansen, L. B. \& Nørskov, J. K. Improved adsorption energetics within density-functional theory using revised Perdew-Burke-Ernzerhof functionals. Phys. Rev. B 59, 7413-7421 (1999).

84. Cheng, T., Wang, L., Merinov, B. V. \& Goddard, W. A. Explanation of dramatic pHdependence of hydrogen binding on noble metal electrode: greatly weakened water adsorption at high pH. J. Am. Chem. Soc. 140, 7787-7790 (2018).

85. Liu, L.-M., Zhang, C., Thornton, G. \& Michaelides, A. Structure and dynamics of liquid water on rutile $\mathrm{TiO}_{2}(110)$. Phys. Rev. B 82, 161415 (2010).

86. Gaigeot, M.-P., Sprik, M. \& Sulpizi, M. Oxide/water interfaces: how the surface chemistry modifies interfacial water properties. J. Phys.: Condens. Matter 24, 124106 (2012).

87. Cheng, J. \& Sprik, M. Alignment of electronic energy levels at electrochemical interfaces. Phys. Chem. Chem. Phys. 14, 11245-11267 (2012).

88. Selcuk, S. \& Selloni, A. Facet-dependent trapping and dynamics of excess electrons at anatase $\mathrm{TiO} 2$ surfaces and aqueous interfaces. Nat. Mater. 15, 1107-1112 (2016).

89. Cheng, J. \& VandeVondele, J. Calculation of electrochemical energy levels in water using the random phase approximation and a double hybrid functional. Phys. Rev. Lett. 116, 086402 (2016).

90. Le, J., lannuzzi, M., Cuesta, A. \& Cheng, J. Determining potentials of zero charge of metal electrodes versus the standard hydrogen electrode from densityfunctional-theory-based molecular dynamics. Phys. Rev. Lett. 119, 016801 (2017).

91. Guo, Z., Ambrosio, F., Chen, W., Gono, P. \& Pasquarello, A. Alignment of redox levels at semiconductor-water interfaces. Chem. Mater. 30, 94-111 (2018).

92. Zhang, C., Hutter, J. \& Sprik, M. Coupling of surface chemistry and electric double layer at $\mathrm{TiO} 2$ electrochemical interfaces. J. Phys. Chem. Lett. 10, 3871-3876 (2019).

93. McCrum, I. T. \& Janik, M. J. pH and alkali cation effects on the Pt cyclic voltammogram explained using density functional theory. J. Phys. Chem. C 120, 457-471 (2016).

94. Schmickler, W. \& Guidelli, R. Ionic adsorption and the surface dipole potential. J. Electroanal. Chem. Interf. Electrochem. 235, 387-392 (1987).

95. Schmickler, W. The surface dipole moment of species adsorbed from a solution. J. Electroanal. Chem. Interf. Electrochem. 249, 25-33 (1988).

96. Garlyyev, B., Xue, S., Watzele, S., Scieszka, D. \& Bandarenka, A. S. Influence of the nature of the alkali metal cations on the electrical double-layer capacitance of model Pt(111) and Au(111) electrodes. J. Phys. Chem. Lett. 9, 1927-1930 (2018).

97. Ringe, S. et al. Understanding cation effects in electrochemical $\mathrm{CO} 2$ reduction. Energy Environ. Sci. 12, 3001-3014 (2019).

98. Schmickler, W. \& Trasatti, S. Comment on trends in the exchange current for hydrogen evolution [J. Electrochem. Soc., 152, J23 (2005)]. J. Electrochem. Soc. 153, L31-L32 (2006).

99. Bouzid, A. \& Pasquarello, A. Atomic-scale simulation of electrochemical processes at electrode/water interfaces under referenced bias potential. J. Phys. Chem. Lett. 9, 1880-1884 (2018).

100. Giannozzi, P. et al. QUANTUM ESPRESSO: a modular and open-source software project for quantum simulations of materials. J. Phys. 21, 395502 (2009).

101. Perdew, J. P., Burke, K. \& Ernzerhof, M. Generalized gradient approximation made simple. Phys. Rev. Lett. 77, 3865 (1996).
102. Prandini, G., Marrazzo, A., Castelli, I. E., Mounet, N. \& Marzari, N. Precision and efficiency in solid-state pseudopotential calculations. npj Comput. Mater. 4, 72 (2018).

103. Marzari, N., Vanderbilt, D., De Vita, A. \& Payne, M. C. Thermal contraction and disordering of the Al(110) surface. Phys. Rev. Lett. 82, 3296-3299 (1999).

104. Dupont, C., Andreussi, O. \& Marzari, N. Self-consistent continuum solvation (SCCS): the case of charged systems. J. Chem. Phys. 139, 214110 (2013).

105. Andreussi, O. \& Marzari, N. Electrostatics of solvated systems in periodic boundary conditions. Phys. Rev. B 90, 245101 (2014).

106. Jülich Supercomputing Centre. JUWELS: Modular Tier-0/1 Supercomputer at the Jülich Supercomputing Centre. J. Large-Scale Res. Facil. 5, A135 (2019).

\section{ACKNOWLEDGEMENTS}

We acknowledge partial financial support from the Swiss National Science Foundation (SNSF) through the NCCR MARVEL, the EU through the MAX CoE for einfrastructure and the Marie Sklodowska-Curie grant agreement No 754462, as well as the German Research Foundation (DFG) CoE e-conversion EXC 2089/1. This work was supported by a grant from the Swiss National Supercomputing Centre (CSCS) under project ID s836 and the computing facilities of SCITAS, EPFL. Furthermore, the authors gratefully acknowledge the Gauss Centre for Supercomputing e.V. (www. gauss-centre.eu) by providing computing time through the John von Neumann Institute for Computing (NIC) on the GCS Supercomputer JUWELS ${ }^{106}$ at Juelich Supercomputing Centre (JSC). Open access funding provided by Projekt DEAL.

\section{AUTHOR CONTRIBUTIONS}

N.G.H. executed the presented simulations. All authors discussed the results and contributed to the paper. The paper was reviewed by all authors.

\section{COMPETING INTERESTS}

The authors declare no competing interests.

\section{ADDITIONAL INFORMATION}

Supplementary information is available for this paper at https://doi.org/10.1038/ s41524-020-00394-4.

Correspondence and requests for materials should be addressed to N.G.H.

Reprints and permission information is available at http://www.nature.com/ reprints

Publisher's note Springer Nature remains neutral with regard to jurisdictional claims in published maps and institutional affiliations.

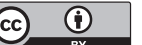

Open Access This article is licensed under a Creative Commons Attribution 4.0 International License, which permits use, sharing, adaptation, distribution and reproduction in any medium or format, as long as you give appropriate credit to the original author(s) and the source, provide a link to the Creative Commons license, and indicate if changes were made. The images or other third party material in this article are included in the article's Creative Commons license, unless indicated otherwise in a credit line to the material. If material is not included in the article's Creative Commons license and your intended use is not permitted by statutory regulation or exceeds the permitted use, you will need to obtain permission directly from the copyright holder. To view a copy of this license, visit http://creativecommons. org/licenses/by/4.0/.

(c) The Author(s) 2020 